\title{
International Forum on ICH Education and Formation as a Scholarly Discipline Held at Beijing Normal University
}

\author{
Jia Zhijie ${ }^{1}$ \\ Beijing Normal University \\ ${ }^{1}$ No. 19, Xinjiekouwi St. \\ Haidian District \\ Beijing \\ 100875 \\ People's Republic of China \\ 202031080054@mail.bnu.edu.cn
}

This project report was accepted for publication on April 1, 2021.

\begin{abstract}
This project report describes the International Forum on $\mathrm{ICH}$ Education and Formation as a Scholarly Discipline held at Beijing Normal University on December 5-6, 2020. The author reviews the key topics and themes explored during the forum, which was focused on the development of educational programs and curriculum related to intangible cultural heritage $(\mathrm{ICH})$.
\end{abstract}

Keywords

cultural policy; disciplines; higher education; intangible cultural heritage; China.

Competing Interests

The author declares no competing interests.

License

Creative Commons CC BY-NC-SA 4.0

Museum Anthropology Review 


\title{
International Forum on ICH Education and Formation as a Scholarly Discipline Held at Beijing Normal University
}

\author{
Jia Zhijie ${ }^{1}$ \\ Beijing Normal University
}

\begin{abstract}
This project report describes the International Forum on ICH Education and Formation as a Scholarly Discipline held at Beijing Normal University on December 5-6, 2020. The author reviews the key topics and themes explored during the forum, which was focused on the development of educational programs and curriculum related to intangible cultural heritage (ICH).
\end{abstract}

[Keywords: cultural policy; disciplines; higher education; intangible cultural heritage; China.]

\footnotetext{
$\mathrm{O}$
} n December 5-6 2020, the International Forum on ICH Education and Formation as a Scholarly Discipline was held at the Beijing Normal University. The conference was organized by the Research and Development Center for ICH as well as the Department of Folklore Studies, both at Beijing Normal University (BNU) and was sponsored by the School of Chinese Language and Literature at BNU and the Cultural Heritage and Cultural Transmission: A Leading-Edge Discipline-Building Project in Beijing Universities initiative. The conference was held in both in-person and online formats, with forty-seven scholars, policy makers, cultural practitioners in management positions, and traditional bearers taking part in the discussions. The group of participants included seven international scholars from the United States, Japan and South Korea (Figures 1-2).

In a keynote speech at the conference, Wang Chenyang, a primary inspector of the Department of Intangible Cultural Heritage at the Ministry of Culture and Tourism of the People's Republic of China, pointed out that the participation of colleges and universities and the protection of intangible cultural heritage mutually impact and promote each other. Taking the specific practice of the Training and Studying Project for Bearers of ICH in China as an example, he argued that we should strengthen the training of ICH teachers, develop ICH safeguarding curriculum in vocational colleges, and strengthen the development of an ICH discipline system and associated academic degrees. Lisa Gilman, Editor-in-Chief of the Journal of American Folklore and a Professor of Folklore at George Mason University, drew upon on her long-term fieldwork in southern Africa and her teaching practice at American universities, to suggest that ICH work in higher education can span instruction related to traditional arts and culture through coursework and by providing additional training for cultural workers to effectively and ethically develop strategies for cultural conservation. She pointed out that scholars also need to challenge existing definitions and disciplinary structures to dismantle the colonial legacies that are engrained in institutional structures, 


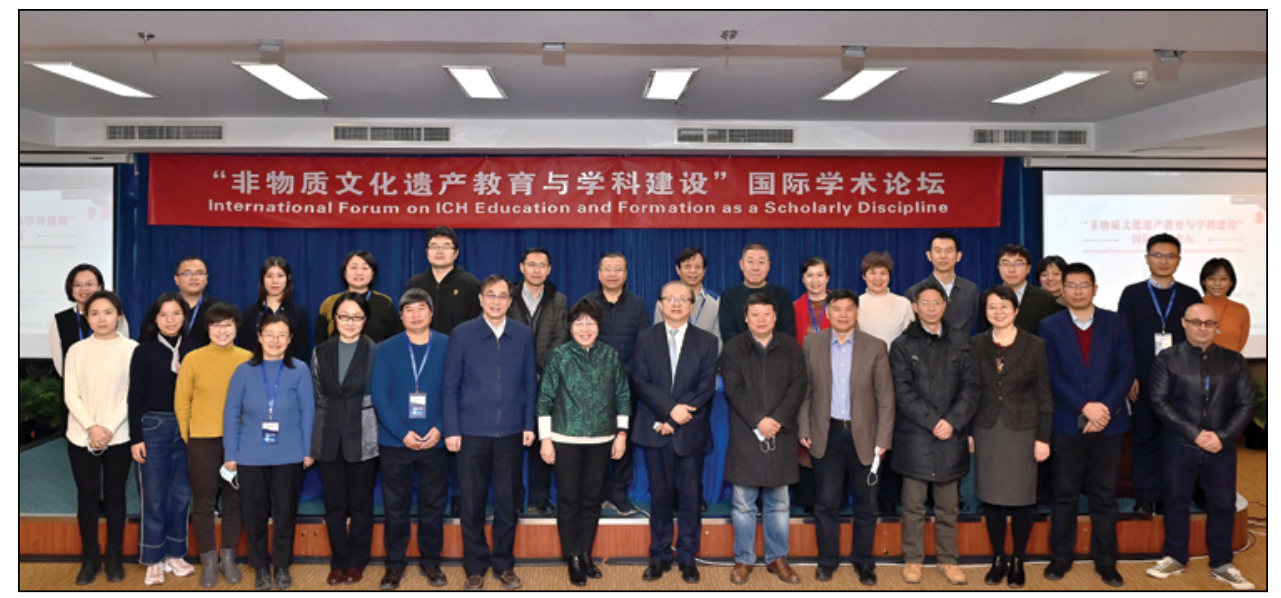

Figure 1. Group Photo of Some In-Person Participants at the International Forum. December 5 , 2020. Photo by Song Jingjing. ${ }^{2}$

curricula, and the reality of who gets to be a student or professor. Building on Transforming Our World: The 2030 Agenda for Sustainable Development adopted by the United Nations (UN) in 2015, Bamo Qubumo, a folklorist at the Chinese Academy of Social Sciences, focused on the cross-cutting concerns between the UN sustainable development goals and the transmission of, and education about, ICH. Bamo stressed as well the further linking of heritage education with UNESCO's recent documents related to the international cooperation mechanism for ICH safeguarding, so as to promote the discussion of the possible approaches to ICH safeguarding in the field of cross-sectoral policymaking.

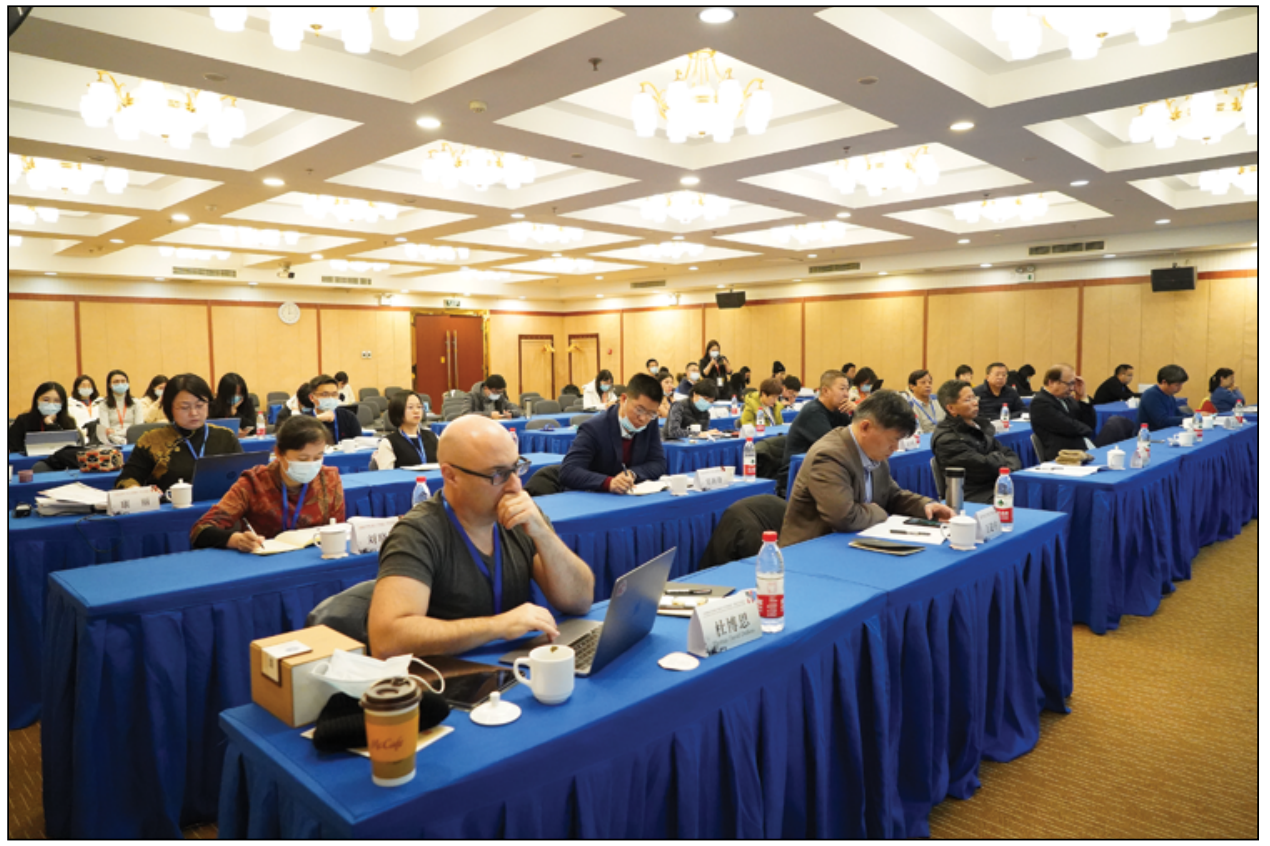

Figure 2. A session of the international forum. December 5, 2020. Photo by Song Jingjing. 
During the two-day conference, participants engaged in lively discussions on the following five main topics:

\section{The Concept and Practice of ICH Education}

Education is an esential part of the measures for the ICH safeguarding. In the Convention for the Safeguarding of Intangible Cultural Heritage (2003), the United Nations Educational, Scientific and Cultural Organization states that States Parties shall work on the education, promotion, and capacity building of ICH safeguarding in a variety of ways. As one of the parties to the convention, China regards education as an important form of ICH safeguarding. During the conference, Gao Bingzhong analyzed the concept of "ICH education", and presented a process of bridging the gap between inheritance of ICH and modern education, which has witnessed profound social and political transformation. Yang Lihui, Huang Yonglin, and others noted that the practice of ICH education based in folklore and folk literature studies has a long history in China. Such work started before the concept of ICH was introduced. From the perspectives of "consciousness-raising" and pedagogy, scholars such as Kang Li, Wang Jiahua and Ma Zhiyao each respectively reflected on educational practice with the theme of gender equality and power transfer within the gender system, expounding on the feasibility and limitation of formal school education in promoting ICH education. Taking the Mongolian Chang Diao talent training project as an example, Se Yin pointed out that ICH training programs can lay a solid foundation for the practice of ICH safeguarding and education.

\section{The Feasibility of the Development of an ICH Discipline}

Recently, the Ministry of Education of the People' Republic of China expressed its support for universities to set up relevant programs for undergraduates, encouraging higher education institutes to strengthen ICH-related majors and to develop curriculum for ICH-related programs. At the opening ceremony of the conference, Chen Li, Vice-Chancellor of Beijing Normal University, who is a specialist in education and pedagogy, said that the study of ICH is a new, growing area for the expansion of university programs in the context of globalization and modernization, and that the educational and academic sectors should actively respond to, and engage in, this pressing social demand. In doing so, the university will improve the capacity of its disciplines to serve society and the country's major strategic development. In a pre-recorded video, Feng Jicai also said that more attention should be paid to the scientific protection of ICH. He grounded his remarks on the basis of experience carrying out a comprehensive investigation of the identification and safeguarding of China's ICH and carrying out survey, identification, and repair of traditional villages. He believes that development of ICH programs and related training are the premise of scientific protection. He expressed his hope that the academic community can work together to actively explore the issue of ICH safeguarding and thus forge ahead.

During the conference, many scholars discussed the topic of the development of ICH-centered university programs. For example, Huang Yonglin, Song Junhua, Zhu Gang, and other scholars discussed the feasibility and necessity of the development of this kind of university programs from theoretical and practical aspects. They and others expressed the opinion that the development of the ICH programs has adapted to the shifting of the research focus of Chinese folk culture studies and 
continuing disciplinary development. Shifts in Chinese folk culture studies and the field's development has occurred not only under the influence of global trends, but also is in response to China's political, social, historical, and cultural transformation in the modernization process. These societal and disciplinary contexts are of significance to ICH safeguarding. Zhang Juwen believes that, as a practical rather than theoretical study, ICH can be viewed as an integrated subject to promote general education. However, it is difficult for it to stand in the existing disciplinary system as a specialized research-based discipline. He Bin, Zheng Ranhe, Pang Jianchun, and other scholars have reviewed and reflected on the development of ICH university programs and practice in Japan and South Korea, which can be helpful examples for similar practice in China.

\section{The Role of Colleges and Universities in ICH Education.}

As an important setting for cultural education and talent cultivation, colleges and universities are obligated to promote the education and disciplinary development of ICH policy, practice, and scholarship. Most of the scholars at the conference focused on the role of universities in ICH safeguarding, attending to the significance, methods, and effectiveness of ICH education in higher education. Zheng Tuyou, Huang Jingchun, Zhou Fuyan, and other scholars discussed how colleges and universities, in ICH-related teaching, should set up ICH safeguarding courses, teach ICH-related knowledge, cultivate students' awareness of ICH safeguarding, improve students' capabilities to carry out ICH safeguarding work, and cultivate and train ICH bearers. But they should be mindful of not erasing the uniqueness and individuality of ICH and should work on preventing ICH from being standardized and over-commercialized. They should also fully consider the consequences of ICH being objectified and being produced as formal knowledge. Song Junhua, Ji Zhongyang, Wang Yao, Wu Xinfeng, and some other scholars shared their experiences in ICH education at Sun Yat-sen University, Nanjing Agricultural University, and Beijing Normal University respectively. Zhang Bo, Tang Lulu and some other scholars, from theoretical perspectives, discussed the coupling between the mission of higher education institutes and ICH safeguarding, the role of universities in the transmission and innovation of ICH, and the role of university researchers as cultural brokers.

\section{The Subject of ICH Education}

In line with the folklorists' emphasis on the subject of cultural practice, a big focus of the conference was the subject of ICH education. For example, Wan Jianzhong thinks that the local cultural elites conducted their work at the most primary-level of the ICH safeguarding, and undoubtedly their greater voice for ICH Safeguarding should be secured. In the past, they were academic "others" and the interviewees in interviews. However, in the context of the discipline construction of ICH, they play a leading role. From the perspective of legal rights and the obligations of tradition bearers, Zhou Xing reflected on the possible change of context for the ICH as well as the transformation of ICH itself that can be brought about by the multiple practices of ICH education. He thought that ICH education provides convenience for bearers to perform part of their legal obligations, and meanwhile raise the motivation of bearers for adapting the intangible cultural heritage that should be protected and maintained by them (or which lead to the change of cultural heritage). Mao Qiaohui analyzed the differences in meaning interpretation and cultural expression between scholars and ICH artists 
and warned scholars to be aware of the complicated relationship between themselves and ICH artists in the practice of ICH safeguarding. The conference also invited four designated ICH bearers to attend the meeting. They are Zhong Liansheng, Ha Yiqi, Chi Shangming, and Liu Xiaodi. They all talked about the issue of multiple subjects in ICH education and pointed out that the responsibilities and obligations of the designated bearers should be further clarified with the goal of more clearly identifying the bearers as the subject of ICH education.

\section{The Critique of ICH}

Critical thinking about ICH related issues should be an important part of the ICH safeguarding. China has carried forward the practice of ICH safeguarding over a relatively long period of time. However, critical thinking about ICH still seems to be insufficient. This is one of the issues that the conference was trying to push forward. Chen Lianshan, Wang Xiaokui, Peng Mu, Yue Yongyi, Zhu Gang, and some other participants reflected on of the concept of ICH. Considering the practice of ICH protection in both China and abroad, they argued that the concept of ICH conceals a complicated relationship of cultural power. They pointed out that the administrative recognition of ICH is not a closed premise without need of further questioning. The meaning and value of ICH as recognized by institutions of cultural administration should not be directly used as the conclusion of academic research. Likewise, the critical analysis process also should not be unreflexively transformed into the argumentation and deduction of this conclusion. The essence of ICH could be explored academically and rationally. Participating scholars such as Xiao Fang, Zhang Shishan, Gao Hehong, and Thomas Dubois, examining Chinese practices of ICH protection from various angles, discussed the ways of integrating ICH into modern life, and the predicament of identifying ICH in public cultural activities, the diverse modes of ICH transmission, and the importance of experiencing ICH in-person.

At the closing ceremony of the conference, Wan Jianzhong pointed out that ICH education is interdisciplinary, comprehensive, and includes multiple subjects. The development of an ICH-centered discipline can provide an opportunity for the reform of the current disciplinary system and for innovation in pedagogy. In these contexts universities have a lot to offer. Yang Lihui summarized the discussions on the above five themes of the conference and pointed out that, since we have already seen significant achievement in Chinese ICH safeguarding, it is important to strengthen the educational system of ICH and to explore the possibility and necessity of developing related major and discipline. This is also a way for folklore scholars and universities to actively respond to the urgent demand of society. These considerations are relevant not only to the situation in China, but internationally as well.

\section{Acknowledgements}

This article was written with the help of three scholars. Thanks go to Yang Lihui, Jason Baird Jackson and Zhang Lijun for their valuable suggestions, revisions and proofreading. Thanks are also extended to Song Jingjing, a master's candidate at BNU, who provided the photographs for this article. 


\section{Notes}

1. Following Chinese norms, Chinese names appearring in this article are presented with the family name preceeding the personal name. The author's family name is Jia. English names are presented personal name first, family name second.

2. In-person delegates included: Wang Chenyang (Ministry of Culture and Tourism, People's Republic of China), Chen Li (Beijing Normal University), Guo Changbao (Beijing Normal University), Wang Lijun (Beijing Normal University), Wan Jianzhong (Beijing Normal University), Xiao Fang (Beijing Normal University), Chen Lianshan (Peking University), Bamo Qubumo (Chinese Academy of Social Sciences), Yang Lihui (Beijing Normal University), Peng Mu (Beijing Normal University), Wu Xinfeng (Shihezi University), He Shaoya (Beijing Normal University), Thomas Dubois (Beijing Normal University), Zhang Jing (Ministry of Culture and Tourism, People's Republic of China), Ying Yizhi (Ministry of Culture and Tourism , People's Republic of China), Tang Lulu (Beijing Foreign Studies University), Zhang Qingren (Minzu University of China), Li Huixin (Ministry of Culture and Tourism, People's Republic of China), Kang Li (Beijing Normal University), Zhu Pengcheng (Chinese Academy of Social Sciences), Zhang Qian (Beijing Municipal Bureau of Culture and Tourism), Shi Zhenhuai (Beijing Folk Literature and Art Association), Ha Yiqi (Beijing Folk Literature and Art Association), Chi Shangming (Yanqing Yongning Middle School), Liu Xiaodi (National-level ICH inheritor), Zhang Bo (Beijing Union University), Wei Bo (Ministry of Culture and Tourism, People's Republic of China), Wang Jialin (Ministry of Culture and Tourism, People's Republic of China), Jiang Chang(Ministry of Culture and Tourism, the People's Republic of China), Wang Hui (Ministry of Culture and Tourism, People's Republic of China), Wang Yao (Beijing Normal University). Delegate names are not in the order in which they appear in the photograph.

Jia Zhijie is a PhD candidate in the Department of Folklore Studies, School of Chinese Language and Literature at Beijing Normal University. 